\title{
Controlled Synthesis of Silver Nanoparticles Using Double Reductants and Its Voltammetric Characteristics Study
}

\author{
Yubo Duan, Zhihua Xu, Xiaochun Jiang* \\ Key Laboratory for Processing and Application of Catalytic Materials, College of Chemical \\ Engineering, Huanggang Normal University, Huangzhou 438000, PR China
}

Received: $5^{\text {th }}$ November 2015; Revised: $21^{\text {st }}$ January 2016; Accepted: $22^{\text {nd }}$ January 2016

\begin{abstract}
Constructing robust silver nanoparticles (AgNPs) with good shape and dispersibility is of particular interest in analytical applications. Herein, monodispersibility AgNPs with the average size of $20 \mathrm{~nm}$ have been successfully prepared via one-pot method using sodium borohydride and trisodium citrate as co-reductants. The introduction of sodium borohydride greatly accelerated the rate of nucleation, which can effectively solve the problem of broad size distribution. Both shape and dispersibility of AgNPs can be effectively adjusted by simple control of refluxing time or concentrations of the sodium borohydride. We also studied the voltammetric characteristics of the AgNPs using Ag/AgCl solid-state voltammetry. An intense and stable current peak at a low potential could be obtained, which could provide a unique advantage in analytical applications. Copyright (C) 2016 BCREC GROUP. All rights reserved
\end{abstract}

Keywords: AgNPs; double reductants; sodium borohydride; trisodium citrate; solid-state voltammetry

How to Cite: Duan, Y., Xu, Z., Jiang, X. (2016). Controlled Synthesis of Silver Nanoparticles Using Double Reductants and Its Voltammetric Characteristics Study. Bulletin of Chemical Reaction Engineering \& Catalysis, 11 (1): 115-119. (doi:10.9767/bcrec.11.1.433.115-119)

Permalink/DOI: http://dx.doi.org/10.9767/bcrec.11.1.433.115-119

\section{Introduction}

Recently, silver nanoparticles (AgNPs) have received extensive attentions benefiting from their intriguing physicochemical properties, minisize and surface plasmon behaviors. AgNPs have been widely used in many fields including sensors, catalysis, electronics, and surface-enhanced Raman scattering [1-3]. Among them, the excellent electrochemical activity of AgNPs has been extensively applied for the fabrication of sensing platforms owing to the sharper current at milder potential than gold nanoparticle [4]. Especially, the introduc-

* Corresponding Author.

E-mail: yjjjxch@aliyun.com (X. Jiang)

Telp. +86-713-8833611, Fax: +86-713-8833611 ing of solid-state voltammetry further promotes the development of biosensor involving AgNPs because this solid-state voltammetry could produce a sharper oxidation peak with intense peak current and flat baseline as compared to the other voltammetry [5]. Since Ying's group first used this type of voltammetry to detect prostate-specific antigen and DNA oligonucleotide of the avian flu virus H5N1 [6]. This voltammetry has been adopted for ultrasensitive determination of tumor markers [5], Bacillus thuringiensis transgenic sequence [7] and Escherichia coli [8]. These ultrasensitive bioanalysis systems based on solid-state $\mathrm{Ag} / \mathrm{AgCl}$ voltammetry have validated the advantages of using AgNPs in electrochemical bioanalysis. 
The sensitivity and accuracy of the bioanalysis certainly depended on the stability and size of AgNPs used in the biosensor. Only when uniform and stable AgNPs could be fabricated, the sensitive and accurate analysis platform could be established. Hence the synthesis of AgNPs has received considerable attentions in recent years. AgNPs can be prepared by two routes [9]: i.e. physical approach such as evaporation/condensation and laser ablation, and chemical approach in which the silver ions are reduced by reductant such as sodium borohydride and trisodium citrate. The chemical approach is the most frequently applied method because the prepared AgNPs are relatively stable and monodisperse in water or organic solvents.

The mechanism of formation of AgNPs includes two stages: nucleation and growth. The nucleation step requires higher activation energy as compared to the growing step. So when a strong reductant, such as: borohydride, was used, small monodisperse particles could be obtained, but the reaction speed was sharp and difficult to control. When a weak reductant such as trisodium citrate was used, nucleation rate was slow, and the size distribution was wide [9]. Fortunately, researchers found that the nucleation and growth of nanoparticles could be better controlled when the coreduction (i.e., sodium borohydride and trisodium citrate) were employed [10, 11]. This may contribute to the fabrication of uniform and stable AgNPs.

Herein, we fabricated uniform and stable AgNPs via one pot method using sodium borohydride and trisodium citrate as co-reductants. Trisodium citrate was employed as a primary reductant and stabilizer, and slight sodium borohydride was used as strong reductant to accelerate the nucleation progress. That could overcome broad size distribution resulted from the slow nucleation rate when only trisodium citrate was used. The influence including refluxing time and concentrations of the sodium borohydride on the quality of AgNPs was investigated, and the top-quality AgNPs with good monodispersibility and uniform size were successfully obtained when $500 \mu \mathrm{L} 3 \mathrm{mM}$ sodium borohydride was added. In order to examine the electrochemical activity of the prepared AgNPs, the voltammetric characteristics of the AgNPs were also studied. All the AgNPs could provide a strong and intense oxidation peak could obtain at a relatively low potential. It is interesting that among them the top-quality AgNPs displayed the excellent electrochemical performance, which could provide a unique advantage in analytical applications.

\section{Materials and Methods}

\subsection{Materials}

Cysteamine (95.0\%), sodium borohydride (99.0\%), trisodium citrate (98.0\%) and $\mathrm{AgNO}_{3}$ (99.8\%) were bought from Aladdit Chemistry Co. Ltd (Shanghai, China). All chemicals reagent used in this experiment were of analytical grade and all solutions were prepared with ultrapure water.

\subsection{Synthesis and characteristics of AgNPs}

AgNPs were prepared via a facile one pot method by reduction of $\mathrm{AgNO}_{3}$ using sodium borohydride and trisodium citrate as reductants. The detailed procedure can be found in our previous literature [8]. The spectra of AgNPs were obtained by using a UV-VIS Spectrophotometer (Shimadzu, UV-2600). Transmission electron microscopy (TEM) images of the AgNPs were obtained by using a transmission electron microscope (JEOL, JEM-2010) operating at an acceleration voltage of $200 \mathrm{kV}$.

\subsection{Electrode modification and detection}

The gold electrodes were carefully burnished with 1.0, 0.3, and $0.05 \mu \mathrm{m} \mathrm{Al}_{2} \mathrm{O}_{3}$ slurry on a microcloth pad for $5 \mathrm{~min}$, followed by ultrasonic processing in nitric acid (1:1) and ultrapure water, for $5 \mathrm{~min}$, respectively. Subsequently, the electrode was electrochemically cleaned in $1 \mathrm{M}$ of $\mathrm{H}_{2} \mathrm{SO}_{4}$ by Cyclic voltammetry (CV) until a repeatable voltammogram was obtained to remove the contaminants on the electrode surface. The pretreated electrode was soaked in $0.1 \mathrm{M}$ cysteamine water solution for $10 \mathrm{~h}$, followed by cleaning with water, and then immersed in the silver colloid at $4 \circ \mathrm{C}$ for $10 \mathrm{~h}$. Finally the prepared electrode was washed carefully with $10 \mathrm{mM}$ of tris- $\mathrm{HCl} / 0.15 \mathrm{M}$ of $\mathrm{NaCl}$ (pH 8.8).

Cyclic voltammetry (CV) was performed with a CHI 660e electrochemical workstation (Shanghai, China). The experiments were carried out with a three-electrode system. A modified Au electrode with $2 \mathrm{~mm}$-diameter, a platinum wire and an $\mathrm{Ag} / \mathrm{AgCl}$ (saturated $\mathrm{KCl}$ ) electrode were used as the working electrode, counter electrode and reference electrode, respectively. 


\section{Results and Discussion}

\subsection{Synthesis and characteristics of the AgNPs}

Borohydride and trisodium citrate are the most frequently-used reductants, but the strong reducibility of borohydride can result in the out of control of the progress and the weak reducibility of trisodium citrate can result in the wide distribution [9]. In this paper, trisodium citrate was used as the primary reductant and stabilizer, the slight borohydride was used to accelerate the nucleation rate. From Figure 1A, it can be seen that an abundant of silver seeds were produced immediately when borohydride was added (b). The trisodium citrate in solution availably passivated the silver nuclei and prevented their agglomeration. Subsequently, the unspent Ag ions were reduced by trisodium citrate and attached to nuclei. Heated 40 min later, the high quality AgNPs were obtained by the simple and practicable method (f). The prepared AgNPs were confirmed by the TEM and presented in Figure 2B. It illustrates that the AgNPs are spherical particles with good monodispersibility and the average size of $20.0 \mathrm{~nm}$ can be estimated. The size of the AgNPs are more approximate and the shape of them are more similar to sphere than that prepared at the same experiment condition only without adding borohydride (Figure 2A). This result demonstrates that the slight borohydride is very important to synthesize spherical particles with good monodispersibility and narrow distribution.

Certainly, the quantity of the used borohydride was also studied and presented in Figure $1 \mathrm{~B}$. When $500 \mu \mathrm{L} 3 \mathrm{mM}$ sodium borohydride was added, the strongest absorption peak was appeared. When the quantity of the borohydride is too little, that cannot prompt enough silver seeds; while the quantity of the borohydride is too much, too silver seeds prompted by borohydride were unfavourable to form uniform AgNPs [9-11]. The results also were demonstrated by the TEM (unpresented). When the quantity of the borohydride is too little or too much, there are many non-spherical particles and the distribution of the size is broad.

Besides, the fabricated AgNPs were very stable. The AgNPs did not aggregate after placed at room temperature for 30 day. The spectra of AgNPs prepared immediately and stored for 30 day ago were presented in Figure $1 \mathrm{C}$. It was well found that the two spectra coincided very well, which indicated that the AgNPs fabricated by this way were very stable again. The stability of AgNPs maybe resulted from the uniformity of AgNPs and the valid protective effect from trisodium citrate [9-11].

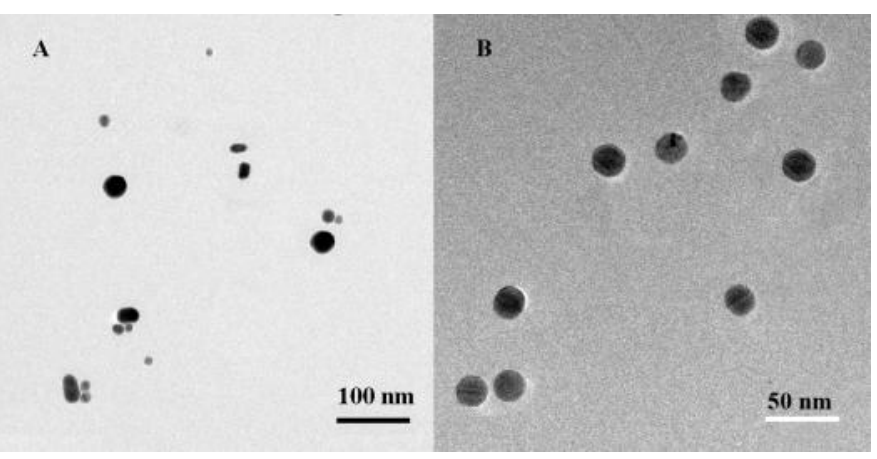

Figure 2. TEM images of AgNPs synthetized by adding different volume of $3 \mathrm{mM}$ sodium borohydride : (A) $0 \mu \mathrm{L}$, (B) $500 \mu \mathrm{L}$
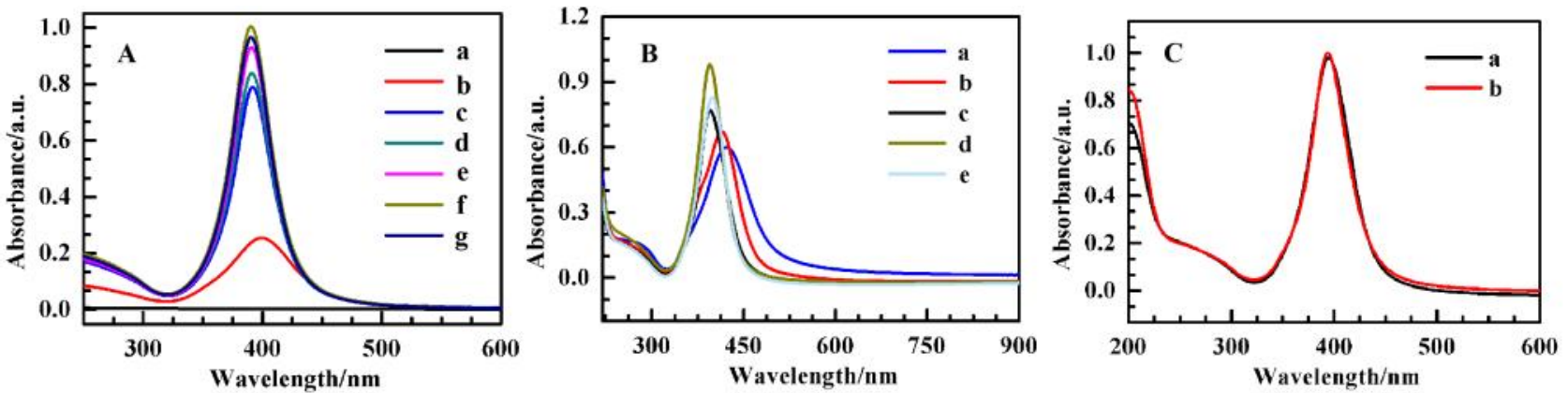

Figure 1. (A) Absorption spectra of reaction solution before (a) and after (b, c, d, e, f and g) adding 500 $\mu \mathrm{L} 3 \mathrm{mM}$ sodium borohydride with heating different time: (b) $0 \mathrm{~min}$, (c) $10 \mathrm{~min}$, (d) $20 \mathrm{~min}$, (e) $30 \mathrm{~min}$, (f) $40 \mathrm{~min}$, (g) $50 \mathrm{~min}$; (B) Absorption spectra of AgNPs synthetized by adding different volume of $3 \mathrm{mM}$ sodium borohydride: (a) $0 \mu \mathrm{L}$, (b) $100 \mu \mathrm{L}$, (c) $300 \mu \mathrm{L}$, (d) $500 \mu \mathrm{L}$, (e) $700 \mu \mathrm{L}$; (C) Absorption spectra of AgNPs obtained at optimum condition stored different times: (a) $0 \mathrm{~d}$, (b) $30 \mathrm{~d}$. 


\subsection{Electrochemical characteristics of the AgNPs}

It is well studied that the sensitivity and accuracy of the biosensor involving AgNPs depended on the stability and size of AgNPs used in the biosensor. Only when controllable and stable AgNPs could be prepared, the sensitive and accrued analysis platform could be established. The electrochemical characteristics of AgNPs prepared were estimated by using $\mathrm{Ag} / \mathrm{AgCl}$ solid-state voltammetry and presented in Figure 3. The all AgNPs with adding different quantity borohydride have strong electrochemical responses, and the most intense current peak was appeared when $500 \mu \mathrm{L} 3 \mathrm{mM}$ sodium borohydride was added. In this progress, AgNPs was oxidized to $\mathrm{Ag}^{+}$, and the generated $\mathrm{Ag}^{+}$formed insoluble $\mathrm{AgCl}$ on the electrode surface when $\mathrm{Cl}^{-}$was existed in solution. Compared with stripping voltammetry, it can lead to a sharper peak at a lower potential, which is far from unwanted oxidative interferences [58]. Besides, it is worth mentioning that the current response of this voltammetry remains unchanged after scanning for 20 cycles. These unique advantages may arouse many researchers to establish bioanalysis method based on $\mathrm{Ag} / \mathrm{AgCl}$ solid-state voltammetry.

\section{Conclusions}

In summary, we have successfully fabricated the controllable and stable AgNPs via a facile one pot method using sodium borohydride and trisodium citrate as reductants. Trisodium citrate was employed as a primary

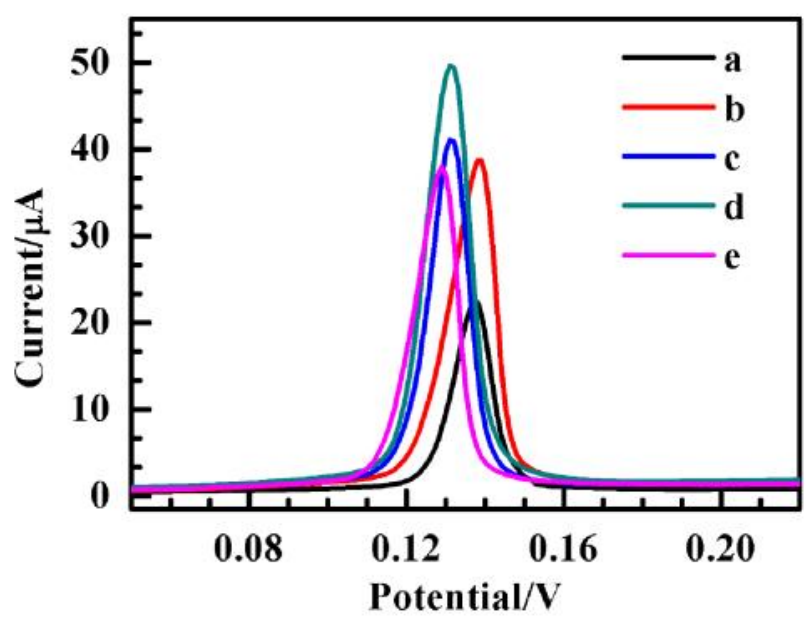

Figure 3. The current response from AgNPs synthetized by adding different volume of $3 \mathrm{mM}$ sodium borohydride: (a) $0 \mu \mathrm{L}$, (b) $100 \mu \mathrm{L}$, (c) 300 $\mu \mathrm{L}$, (d) $500 \mu \mathrm{L}$, (e) $700 \mu \mathrm{L}$. reductant and a stabilizer, and little of sodium borohydride was used as strong reductant to accelerate the nucleation progress. That could overcome the broad size distribution resulted from the slow nucleation rate when only trisodium citrate was used as reductant. The influence including refluxing time and concentrations of the sodium borohydride on the quality of AgNPs was investigated. We found that the AgNPs were spherical particles with good monodispersibility and the average size of that was about $20.0 \mathrm{~nm}$ when $500 \mu \mathrm{L} 3 \mathrm{mM}$ sodium borohydride was added. It is exciting that a strong and intense oxidation peak could obtain at a relatively low potential, which could provide a unique advantage in analytical applications.

\section{Acknowledgments}

We are grateful for the financial support by the Natural Science Foundation of Hubei Province (2014CFB162), the Scientific Research Fund of Hubei Education Department (Q20122701), the Energy Storage and Conversion Innovative Research Team for Distinguished Yong Scholars of Hubei Provincial Department of Education (T201514), Natural Science Foundation of Huanggang Normal University (2014015403) and the Doctoral Scientific Fund Project of Huanggang Normal University (2013031803).

\section{References}

[1] Tolaymat, T.M., El Badawy, A.M., Genaidy, A., Scheckel, K.G., Luxton, T.P., Suidan, M. (2009). An Evidence-based Environmental Perspective of Manufactured Silver Nanoparticle in Syntheses and Applications: A Systematic Review and Critical Appraisal of Peerreviewed Scientific Papers. Science of the Total Environment, 408: 999-1006.

[2] Nair, L.S., Laurencin, C.T., (2007). Silver Nanoparticles: Synthesis and Therapeutic Applications. Journal of Biomedical Nanotechnology, 3: 301-316.

[3] Zhu, Z., Gao, F., Lei, J., Dong, H., Ju, H. (2012). A Competitive Strategy Coupled with Endonuclease-Assisted Target Recycling for DNA Detection Using Silver-NanoparticleTagged Carbon Nanospheres as Labels. Chemistry-A European Journal, 18: 13871-13876.

[4] Cai, H., Xu, Y., Zhu, N.N., He, P.G., Fang, Y.Z. (2002). An electrochemical DNA hybridization detection assay based on a silver nanoparticle label. Analyst, 127: 803-808.

[5] Ting, B.P., Zhang, J., Khan, M., Yang, Y.Y., Ying, J.Y. (2009). The Solid-state $\mathrm{Ag} / \mathrm{AgCl}$ Process as a Highly Sensitive Detection 
Mechanism for an Electrochemical Immunosensor. Chemical Communications 6231-6233.

[6] Zhang, J., Ting, B.P., Jana, N.R., Gao, Z., Ying, J.Y. (2009). Ultrasensitive Electrochemical DNA Biosensors Based on the Detection of a Highly Characteristic Solid-State Process. Small, 5: 1414-1417.

[7] Jiang, X.C., Chen, K., Han, H.Y. (2011). Ultrasensitive Electrochemical Detection of Bacillus Thuringiensis Transgenic Sequence Based on in situ Ag Nanoparticles Aggregates Induced by Biotin-Streptavidin System. Biosensors and Bioelectronics, 28: 464-468.

[8] Jiang, X.C., Chen, K., Wang, J., Shao, K., Shao, F., Fu, T., Lu, D.L., Han, H.Y. (2013). Solid-state Voltammetry Based Electrochemical Immunosensor for Escherichia Coli Using Graphene Oxide-Ag Nanoparticle Composites as Labels. Analyst, 3388-3393.
[9] Abou El-Nour, K.M.M., Eftaiha, A., AlWarthan, A., Ammar, R.A.A. (2010). Synthesis and Applications of Silver Nanoparticles. Arabian Journal of Chemistry, 3: 135-140.

[10] Dong X.Y., Ji X.H., Jing J., Li M.Y., Li J., Yang W.S. (2010). Synthesis of Triangular Silver Nanoprisms by Stepwise Reduction of Sodium Borohydride and Trisodium Citrate. The Journal of Physical Chemistry C, 114: 2070-2074.

[11] Agnihotri, S., Mukherji, S., Mukherji, S. (2014). Size-controlled Silver Nanoparticles Synthesized over the Range 5-100 nm Using the Same Protocol and Their Antibacterial Efficacy. RSC Advance. 4: 3974-3983. 